\title{
Absorption coefficient of doped polycrystalline silicon films in infrared spectrum
}

\author{
Xia Zhang ${ }^{1, a}$ and Dacheng Zhang ${ }^{2, b}$ \\ ${ }^{1}$ Department of Photo-electronics, Communication University of China, Beijing, China \\ ${ }^{2}$ Institute of Micro-Nano electronics, Peking University, Beijing, China \\ zhangxia@cuc.edu.cn, dchzhang@ime.pku.edu.cn
}

\begin{abstract}
Absorption coefficient of materials depends on their extinction coefficient. Based on the equations derived from electromagnetic theory, utilized the value of the measured reflectance and transmittance of the multi-layer films, the extinction coefficient in infrared spectrum of the polycrystalline silicon films with different doped dosage is obtained by means of using the inverse calculation. Thus, the absorption coefficient of the polycrystalline silicon films results. The analysis demonstrates that the absorption coefficient increases with the doped dosages increasing for a given wavelength. This method used for determining absorption coefficient has the advantage of that the measured samples are fabricated simply.
\end{abstract}

\section{Introduction}

As the fabrication method for polycrystalline silicon (Poly-Si) is compatible with integrated circuit processes, it exhibits excellent mechanical performance and the heavily doped Poly-Si is a conductor, Poly-Si film is commonly used for movable structures in the micro sensor base on microelectronics technology. However, the physical properties of thin films are different from that of bulk materials. Therefore, the research on the properties of the doped Poly-Si film is helpful for micro sensor design.

In the paper, based on the equations derived from photonics and electromagnetic theory, utilizing the value of the measured reflectance and transmittance of a layer of plate and the multilayer films, the extinction coefficient in infrared spectrum of a layer of Poly-Si film with a certain doped dosage are obtained by means of using the inverse calculation.

\section{Samples design and fabrication processes}

A practical measurement setup requires millimetres size membranes for a reasonable signal level, to ensure the reflected and transmitted energy to be measured accurately [1]. However, the 
fabrication of the sample in which the free-standing suspended poly-Si film with a thickness of 1$2 \mu \mathrm{m}$ or less satisfies the samples size of $3.5 \times 3.5 \mathrm{~cm}^{2}$ is more complex.

In order to solve the problem brought by the dimensions feature above, a series of samples are designed, fabricated. The prepared samples are as follows:

1 \# sample is the substrate with parameters above.

2\# sample is that a layer of $\mathrm{SiO}_{2}$ film with $300 \mathrm{~nm}$ thickness is grown on the substrate at temperature of $1000^{\circ} \mathrm{C}$ by thermal oxidation, which is used for the implanting isolation in order to control the doped concentration of within the Poly-Si film.

3\# series samples are that a layer of Poly-Si film of $1 \mu \mathrm{m}$ thickness is deposited on $300 \mathrm{~nm}$ thickness $\mathrm{SiO}_{2}$ film with the substrate at temperature of $610^{\circ} \mathrm{C}$ by low pressure chemical vapour deposition (LPCVD). Then, phosphorus-doped is implanted in a certain dosage, and annealing at temperature of $1000^{\circ} \mathrm{C}$. The dosages of sample $3 \#-1,3 \#-2,3 \#-3$ and $3 \#-4$ are $1.0 \times 10^{14} \mathrm{~cm}^{-2}$, $6.0 \times 1014 \mathrm{~cm}^{-2}, 1.6 \times 10^{15} \mathrm{~cm}^{-2}$ and $5.1 \times 10^{15} \mathrm{~cm}^{-2}$, respectively.

\section{Results and analysis}

Applied the equations derived from photonics and electromagnetic theory[2] [3], based on the measured value of the spectral reflectance and transmittance of samples, the extinction coefficient in infrared spectrum of the polycrystalline silicon films with different doped dosages are obtained by means of by the inverse calculation. The calculation results of absorption coefficient and the related parameters of samples are tabulated for a given wavelength of $10 \mu \mathrm{m}$ in Table 1.

Table 1. Sample parameters

\begin{tabular}{|l|l|l|}
\hline Sample number & Implanted dosages $\left(\mathrm{cm}^{-2}\right)$ & Absorption coefficient $\alpha\left(\mathrm{m}^{-1}\right)$ \\
\hline $3 \#-1$ & $1.0 \times 10^{14}$ & 14423.90 \\
\hline $3 \#-2$ & $6.0 \times 10^{14}$ & 44767.61 \\
\hline $3 \#-3$ & $1.6 \times 10^{15}$ & 87947.63 \\
\hline $3 \#-4$ & $5.1 \times 10^{15}$ & 684118.10 \\
\hline
\end{tabular}

Absorption coefficient of materials depends on their extinction coefficient. The extinction coefficient in infrared spectrum of the polycrystalline silicon films with different doped dosages, base on the inverse calculation, are obtained by means of utilizing the measured reflectance and transmittance of a layer of material and multilayer films, and the equations derived from photonics and electromagnetic theory. Therefore, the absorption coefficients of the films result. The analysis demonstrates that the absorption coefficient of the doped polycrystalline silicon films increases with the doped dosages increasing for a given wavelength and there is different absorption coefficient for different wavelength.

\section{Conclusion}

The method is effective that the absorption coefficient of the Poly-Si films is obtained by the inverse calculation. This determination of film parameters has the advantage of that samples are fabricated simply.

\section{References}

[1]Tuohiniemi M, Blomberg M., Gao F., Infrared spectroscopy study of a poly-silicon film for optimizing the boron-implanting dose. Journal of Microelectromechanical Systems. 22 (5), pp. 1207-1212, 2013. 
[2]Sze S M. KWOK K. NG J., Physics of Semiconductor Devices (3rd Edition). pp. 39-41, Xian Jiao Tong University Press, Xian, 2008.

[3]Jinfa T., Peifu G, Xu L, and Haifeng L., Modern Optical Thin Film Technology. pp. 29, Zhejiang University Press, Hangzhou, 2006. 